\title{
Bulging of Posterior Capsule Associated with Choroidal Effusion during Phacoemulsification
}

\author{
Saraswati J $^{1 *}$, Umesh $\mathbf{Y}^{\mathbf{1}}$, Priyanka Sudhakar ${ }^{1}$ and Pradeep Bastola ${ }^{2}$ \\ ${ }^{1}$ General Ophthalmology, Sankara Eye Care, Bangalore, India \\ ${ }^{2}$ Associate Professor in Ophthalmology, Nepal \\ *Corresponding Author: Saraswati J, General Ophthalmology, Sankara Eye Care, \\ Bangalore, India.
}

Received: May 11, 2020

Published: June 10, 2020

(C) All rights are reserved by Saraswati J., et al.

\begin{abstract} sion which is a very rare intraoperative event in an elective case of phacoemulsification.

Keywords: Ciliochoroidal Effusion; Hypertension; Nanophthalmos; Phacoemulsification
\end{abstract}

We report an interesting and a relatively rare case of intraoperative choroidal effusion, during a routine phacoemulsification in a seemingly normal senile cataract, with a bulging of posterior capsule and shallowing of anterior chamber because of choroidal effu-

\section{Introduction}

Shallowing of anterior chamber (AC) and bulging of the posterior capsule with choroidal effusion during intraoperative phacoemulsification are uncommon, even in high risk cases of hypermetropia, nanophthalmos and an underlying systemic hypertension. But very rare in an emmetropic normal eye especially in the modern-day cataract surgery $[1,4]$. In our case report, we discuss in detail, the intraoperative difficulty in such cases, the precautionary measures to be taken and the management of this precarious complication, to achieve an uneventful and more favourable outcome.

A 73-year lady presented with a complain of defective vision in the right eye for 1 year. She was a known diabetic and hypertensive and with a history of cataract surgery in her left eye. A detailed anterior and posterior segment evaluation was done followed by lens star biometry which revealed an axial length of $22.56 \mathrm{~mm}$ which was slightly hypermetropic but considering her age it is in normal side. Post keratometry readings and clinical refraction values, she was undertaken for phacoemulsification surgery with a toric intraocular lens (IOL) implantation under topical (lignocaine gel 2\%) anaesthesia.

The early intraoperative phase was uneventful until cortical aspiration, when the surgeon noticed a sudden anterior chamber (AC) shallowing with anterior bulging of the posterior capsule (PC). Despite liberal use of viscoelastic, the PC bulging and tenting continued, making it difficult to proceed with the surgery. The IOL implantation was thus deferred. Intravenous (IV) mannitol $100 \mathrm{ml}$ stat was given to lower the intra ocular pressure (IOP) on the surgery bed and further avoid dreadful complications.

An immediate ultrasound B scan was done which revealed RCS thickening and minimal fluid in the suprachoroidal space.

The patient was counselled about the condition and on mutual understanding was scheduled for a secondary IOL implantation after 2-weeks. During the 2-week period, the patient was regularly evaluated on the slit lamp for any changes in the anterior or posterior segment and her intra ocular pressure (IOP) record was maintained. patient was started in topical antiglaucoma medication (combination of brimonidine $0.15 \%$ and timolol $0.5 \%$ eyedrop twice a day for 1 week), tapering antibiotic-steroid drops (Moxifloxacin $0.5 \%$ and dexamethasone $0.1 \%$ ) and a cycloplegic (eye drop homatropine) for a week. Following the secondary toric IOL implantation, the patient had a normal visual outcome of $6 / 6$ in the $2^{\text {nd }}$ postoperative week.

\section{Discussion}

Ciliochoroidal effusion is an accumulation of fluid in the potential space between the sclera externally and the choroid and ciliary body internally [1]. In a normal eye, the suprachoroidal space is essentially non-existent because of close apposition of the choroid to the sclera. In pathologic conditions that disrupt the normal ocular fluid dynamics and hydrostatic and oncotic pressure gradients, fluid accumulates in this potential space [2]. The choroid is normally directly next to the sclera, but can get displaced by fluid or blood, leading to a choroidal detachment (separation) [2]. 
It is a common complication following glaucoma surgery. Intraoperative complications and prolonged phacoemulsification time are however have not shown to be risk factors for effusion [3]. The collection of fluid can be serous or haemorrhagic. Though known to occur notably following glaucoma surgery and rarely post or during cataract surgery. This may arise from other intraocular surgeries and many conditions, including inflammatory and infectious diseases, trauma, neoplasms, drug reactions and venous congestion.

Idiopathic causes fall under the umbrella of uveal effusion syndrome, a rare condition usually considered a diagnosis of exclusion [1]. Uveal effusion syndrome is a rare syndrome of idiopathic exudative detachments of choroid, ciliary body and retina, thought to arise from impaired posterior segment drainage usually associated with scleral thickening and vascularity [4]. Though known to occur more postoperatively and rarely intraoperatively, the incidence is observed to be slightly lesser in phacoemulsification as compared to small incision cataract surgery (2.6\%) [5].

Risk factors for developing a choroidal detachment include [6] systemic and ocular abnormality.

Etiologic classification of ciliochoroidal effusion

- Iatrogenic injury

- Medications

- Anterior segment surgery (with or without cyclodialysis)

- Cataract extraction

- Glaucoma filtering procedures

- Iridectomy

- Pan retinal photocoagulation

- Retinal detachment surgery

- Traumatic injury

- Accidental ocular perforation

- Blunt trauma

- Inflammation

- Episcleritis

- Nonspecific vasculitis occlusive vasculitis

- Orbital pseudotumor

- Rheumatoid arthritis

- Scleritis

- Uveitis

- Vascular causes

- Valsalva maneuver

- Carotid-cavernous sinus fistula

- Dural-sinus fistula

- Hypertension

- Hypoproteinaemia

\section{- Malignancy}

- Metastatic carcinoma to the choroid

- Leukaemia

\section{- Primary scleral abnormality}

- Nanophthalmos

- Uveal effusion syndrome

- Idiopathic ciliochoroidal effusion.

Since acute choroidal effusion is a rare condition with signs like expulsive choroidal haemorrhage. It has been postulated by Maumenee and Schwartz that expulsive haemorrhage begin with an acute serous choroidal effusion, the mechanism which was laid for this is the detachment of the posterior choroid and the sudden stretching of the posterior ciliary vessels causing them to rupture. Hence recognising and understanding the signs plays an important role [11].

As an intraoperative complication, it can present initially with a bulging of the posterior capsule with minimal to no shallowing of anterior chamber with or without rise in intraocular pressure, posterior capsular rent, and expulsive choroidal haemorrhage.

Large effusions may cause refractive changes owing to anterior displacement of the lens-iris diaphragm and resulting in myopia, or they may cause significant reduction in visual acuity by encroachment into the visual axis. Patients may experience an absolute scotoma at the site of effusion [6]. Therefore, caution should be taken in each step if surgeons finds bulging of posterior capsule, anterior chamber shallow, rise in intraocular pressure.

Going by the golden words "early identification of the red flag signs is vital".

Characteristic clinical features of a ciliochoroidal effusion [6]

- Choroidal edema

- Shallow anterior chamber

- Low intraocular pressure

- Visualization of the ora serrata without scleral depression

- Non-rhegmatogenous retinal detachment with shifting sub-retinal fluid

- Verhoeff's streaks [7]

- Annular, lobular, or flat Ophthalmoscopic appearance

- $\quad$ Hagen's sign [8]. 


\section{Management}

Diagnosis: Clinical features assessment plus

1. Fluorescein angiography (FAG) - Rules out other causes of exudative retinal detachment.

2. ICG, Optical Coherence Tomography (OCT)

3. Ultrasonography (USG) B scan

4. Ultrasound Biomicroscope - Measures scleral thickness 2 - 3 $\mathrm{mm}$ posterior to the scleral spur (normal scleral thickness).

\section{Treatment}

Treatment of choroidal effusion depends on severity of the fluid collection, in case of minimal effusion with normal IOP, wait and watch by observation is the preferred way. In moderate to severe effusion the patient should be put on drugs to lower intraocular pressure, steroids and may need surgical drainage [9].

In this case, we suspect the cause of choroidal effusion to be attributed to hypertensive status of the patient, which correlates very well to a study by Suto C., et al. [10] who also reported a rare case of choroidal effusion in an uncomplicated cataract surgery in a hypertensive patient on antihypertensive medications [10].

\section{Conclusion}

Intraoperative choroidal effusion, though, a rare and dangerous complication, one must anticipate this in the presence of certain pre-existing risk factors and subsequently do a thorough ophthalmic and systemic evaluation with an emphasis on biometry.

\section{Bibliography}

1. Speath EP and deLong P. "Detachment of the Choroid A Clinical and Histopathological Analysis". Archives of Ophthalmology 32 (1944): 217.

2. Weiter JJ and Ernest JJ. "Anatomy of Choroidal Vasculature". American Journal of Ophthalmology 78.4 (1974): 583-590.

3. Anvesh C Reddy and Sarwat Salim. Choroidal Effusion, American Academy Ophthalmology Clinical Pearls. Eyenet Magazine (2012).

4. Khalid Sabti., et al. "Uveal Effusion After Cataract Surgery: An Echographic Study”. Ophthalmology 108.1: 100-103.

5. Gass JD. "Uveal effusion syndrome. A new hypothesis concerning pathogenesis and technique of surgical treatment". Retina 3.3 (1983): 159-163.
6. RR Shah., et al. "Flat anterior chamber and choroidal detachment in aphakia. Study of cataract extractions". British Journal of Ophthalmology 55.1 (1971): 48-49.

7. Verhoeff FH. "The nature and origin of the pigmented streaks caused by separation of the choroid". Journal of the American Medical Association 97.25 (1931): 1873-1877.

8. Hagen S. "Die serose postoperative Choroideallablosung und ihre pathogenese". Klinische Monatsblatter fur Augenheilkunde 66 (1921): 121.

9. WuDunn D., et al. "Surgical Drainage of Choroidal Effusions Following Glaucoma Surgery". Journal of Glaucoma 14.2 (2005): 103-108.

10. Suto C., et al. "Choroidal detachment after uncomplicated small incision cataract surgery". Case Reports in Ophthalmology 3.2 (2012): 175-179.

11. Payne JW., et al. "Expulsive haemorrhage: its incidence in cataract surgery and a report of four bilateral cases". Transactions of the American Ophthalmological Society 83 (1985): 181-204.

\section{Assets from publication with us}

- Prompt Acknowledgement after receiving the article

- Thorough Double blinded peer review

- Rapid Publication

- Issue of Publication Certificate

- High visibility of your Published work

Website: www.actascientific.com/

Submit Article: www.actascientific.com/submission.php

Email us: editor@actascientific.com

Contact us: +919182824667 